\title{
The biometrical genetics of competitive parameters in Drosophila melanogaster
}

\author{
Mortaza Hemmat and \\ Paul Eggleston
}

\author{
Department of Genetics and Microbiology, \\ University of Liverpool, P.O.Box 147, \\ Liverpool L69 3BX, U.K.
}

Despite the importance of competition as an evolutionary determinant in natural populations there have been few studies of the genetical control of competitive ability. Here, we report the results of a biometrical analysis of four continuously varying traits which, between them, describe the competitive interactions in mixed cultures of Drosophila melanogaster. The analysis involved the parental, $F_{1}, F_{2}$ and backcross generations (including all reciprocals) derived

from crosses between two highly inbred lines isolated from the Texas population of $D$. melanogaster. The competitive performance of each genotype in monoculture and in duoculture with a phenotypically distinct tester were assessed using a yield-density regression analysis. Appropriate genetic models were fitted using a variance weighted least squares procedure and the resulting genetic components of the generation means used to define the genetical architecture of competition. Of the four competitive parameters investigated here the $e$-value, which describes the competitive performance of the indicator genotype at a fixed reference density, was found to be determined by simple additive genetic effects with no evidence of significant dominance. Conversely, competitive performance in monoculture (intra-genotypic competition) did display a significant net dominance component and the observed values in the $F_{1}$ and parental generations indicated some degree of heterosis. Of the two competitive parameters determining performance in duoculture (inter-genotypic sensitivity and inter-genotypic pressure) the former was found to have a complex genetic determination involving not only additive and dominance components of the progeny's own genotype but also dominance components of the $F_{1}$ maternal genotypes. There were also additive-dominance and dominance-dominance non-allelic interactions. Heterosis was evident, determined both by the progeny's own genotype and by one of the $F_{1}$ maternal genotypes. All dominance and heterosis was directed towards reduced inter-genotypic sensitivity or, in other words, superior competitive ability. The analysis of maternal effect components for inter-genotypic competitive pressure could not be accommodated for reasons described in the text, although the data provided evidence for their involvement. The fitting of a simplified model revealed significant additive and dominance components of similar magnitude together with heterosis determined by the progeny's own genotype. There was no evidence of non-allelic interaction. As before all dominance and heterosis was directed towards superior competitive ability (i.e., increased inter-genotypic pressure). Throughout the experiment, there was no evidence for sex-linkage in the determination of competitive parameters. This is thought to be a prerequisite for stability of the sex ratio in the intense competitive environment of natural populations. Possible interpretations of the genetical architecture of competition are discussed in the light of these results.

\section{INTRODUCTION}

Despite extensive investigation of competition and competitive ability, little attention has been given to the nature of the underlying genetical control. Those studies which record the variation present between inbred lines (Eggleston, 1985) or $F_{1}$ hybrids (Mather and Caligari, 1983), provide little insight into the genetic architecture of competition. Furthermore, those experiments which have provided more genetic information (Mather and Cooke, 1962 in Drosophila and Lerner and Ho,
1961 in Tribolium) often have not exercised sufficient control over the experimental conditions. However, all of these studies have hinted at the presence of high levels of heterosis and epistasis, both of which may be expected for a character so closely related to fitness and possibly involving a large part of the genome (Mather, 1983).

Recent improvements in the analysis of competition through yield-density regression analysis (Suehiro and Ogawa, 1980; Mather and Caligari, 1981; Wright, 1981; Spitters, 1983; Watkinson, 1984) have made it possible to represent competi- 
tive abilities by a series of linear parameters. This refinement has emphasised the need for careful control over the environmental conditions necessary for the measurement of competition, such as density and the amount of food. The form of analysis employed here (developed by Mather and Caligari, 1981) yields the following competitive parameters. First, the absolute performance of a genotype at a standard reference density ( $e$ value); secondly, the effect of monoculture density on performance (intra-genotypic competition); thirdly, the influence of a genotype on the performance of other genotypes (inter-genotypic pressure) and fourthly, the response of a genotype to the pressure exerted by associate genotypes (intergenotypic sensitivity). Further analysis yields two related parameters which have been denoted aggression $(a)$ and response $(r)$ (Mather and Caligari, 1983).

Recent investigations using these competitive parameters have improved our understanding of their genetical control. For example, Hemmat and Eggleston (1988a) showed that aggression and response may be adjusted by the selection of particular groups of genes, although the two components do not behave entirely independently. In a chromosome assay experiment using $D$. melanogaster substitution lines, Caligari and Mather (1988) found that all three major chromosomes were involved in the determination of competitive ability. However, the relative importance of these chromosomes varied among the competitive parameters. They showed that genes carried on chromosome III affected both aggression and response, whereas those on chromosome II primarily affected aggression only. The involvement of chromosome I appeared to be limited to an interaction with chromosome II, with respect to the control of response.

Further experiments by de Miranda and Eggleston (1988c) revealed high levels of heterosis for inter-genotypic pressure and slight tendencies towards heterosis for the $e$-value and intergenotypic sensitivity. Intra-genotypic competitive effects appeared to be mostly under additive genetic control, with a minor involvement of additive $\times$ additive gene interactions. Other studies on yield have also shown the involvement of heterosis, for example Barnes $(1968 a, b)$ concerning the yield of adult flies in the $F_{1}, F_{2}$ and backcross generations derived from a cross between two inbred lines of D. melanogaster. Furthermore, Bonnier (1961) has demonstrated that the proportion of freshly hatched larvae that reached eclosion was higher in the hybrids between three unrelated wild- type stocks of $D$. melanogaster than in the stocks themselves. However, Barnes (1968b) described the involvement not only of heterosis determined by the progeny's own genotype, but also that determined by the maternal genotype.

Conventional genetic analyses (Mather and Jinks, 1982; Falconer, 1986) define the genetic control of quantitative characters in terms of the additive and dominance deviations at the many loci influencing the character. Different generations of crosses have their own expectation of mean performance and variance according to the expected genetic constitution of the progeny. In this respect the segregating generations $\left(F_{2}, F_{3}\right.$ and backcrosses) are particularly useful. Based on this type of genetic analysis, the present experiment was designed to investigate the genetical architecture of a range of competitive parameters.

\section{EXPERIMENTAL DETAILS}

The two parental genotypes used in this study were highly inbred lines derived from the Texas population of $D$. melanogaster. The origin of this material has been described by Linney et al. (1971). These two genotypes were chosen following a preliminary experiment which was designed to compare a range of inbred lines isolated from the Texas population of D. melanogaster (Eggleston, 1985). The results of this experiment showed that T1 and T25 represented the extremes of the phenotypic variation in competitive ability observed between those inbred lines and probably also within this population. These two lines could, therefore, be assumed to differ for the majority of the alleles which determine competitive ability and to encompass an appropriate gene pool with which to approach this investigation. In addition, a highly inbred line marked with the body colour mutation $y^{2}$ was chosen as the tester genotype, in order to estimate four competitive parameters (e-value, intragenotypic competition, inter-genotypic sensitivity and inter-genotypic pressure).

Parental, $F_{1}, F_{2}$ and backcross generations, including all reciprocals, were raised so as to ensure an adequate supply of eggs from each generation for the initiation of the experiment. In order to minimise the environmental variation, it was necessary to test all segregating and nonsegregating generations against the $y^{2}$ genotype simultaneously. Although the segregating generations clearly involve a mixture of genotypes, previous studies have shown that they can be considered as a single genotype, in exactly the same 
way as the non-segregating generations, when using this experimental design (Eggleston, 1985). The resulting parameters simply reflect the average performance of the genotypes within each segregating generation. Eggs were collected from each genotype and seeded into monoculture and duoculture tubes along with eggs of the tester genotype $y^{2}$, where necessary, as described by Hemmat and Eggleston (1988a). In addition, a monoculture density series for the tester $\left(y^{2}\right)$ was raised simultaneously with the rest of the cultures. All monocultures and duocultures were raised in duplicate to provide an estimate of the sampling variation. Each vial contained $45 \mathrm{mg}$ yeast (Sigma YSC-2) and all cultures were individually randomised and incubated at $25 \pm 0 \cdot 5^{\circ} \mathrm{C}$. The competitive character investigated was $P_{a}$, the proportion of eggs which successfully develop into adults, converted to angles as described by Fisher and Yates (1963). The data were analysed using the regression analysis of Mather and Calgari (1981). This yielded four competitive parameters, namely, the $e$-value, which represents the absolute performance of each genotype at the reference density of one hundred and twenty eggs per culture; the intra-genotypic competitive value, representing the competitive effect in monoculture; the intergenotypic pressure exerted by the associate genotype (wild type) on indicator genotype $\left(y^{2}\right)$ and the inter-genotypic sensitivity of the indicator genotype (wild type) to the pressure exerted by the associate genotype $\left(y^{2}\right)$. The use of a yielddensity regression analysis and the presence of multiple individuals in any one culture means that the estimated parameters are representative of the genotype as a whole and not of individuals within the genotype.

All of the models employed here for the genetic analysis of competitive parameters are based on the notation of Fisher et al., (1932). Thus, individuals with the genotypes $A A, A a$ and $a a$ have quantitative phenotypes $+\mathrm{d}, \mathrm{h}$ and $-\mathrm{d}$ respectively and the origin of measurement is the mid-homozygote $(\mathrm{m})$. These genetic components

Table 1 Estimates of the competitive parameters (upper values) and their respective error variances (lower values) for parental, $\mathrm{F}_{1}, \mathrm{~F}_{2}$ and backcross generations. $e$ refers to the $e$-values, $C_{x x}$ to intra-genotypic competition, $C_{x y}$ to inter-genotypic sensitivity and $C_{v x}$ to inter-genotypic pressure. (1) and (2) refer to the parental inbred lines T25 and T1 respectively. In all cases T25 was taken to be the higher scoring or increasing parent and T1 the lower scoring or decreasing parent. Using standard notation, the genotype given first in each cross represents the maternal parent

\begin{tabular}{|c|c|c|c|c|c|}
\hline Generation & Mating & $e$-value & $C_{x x}$ & $C_{x y}$ & $C_{y x}$ \\
\hline \multirow[t]{2}{*}{$P_{1}(T 25)$} & \multirow[t]{2}{*}{$(1 \times 1)$} & $51 \cdot 0946$ & 0.273529 & 0.082071 & 0.434286 \\
\hline & & $10 \cdot 5281$ & 0.003700 & 0.003103 & 0.005499 \\
\hline \multirow{2}{*}{$P_{2}(T 1)$} & \multirow[t]{2}{*}{$(2 \times 2)$} & $39 \cdot 1331$ & 0.357075 & $0 \cdot 344964$ & $0 \cdot 160595$ \\
\hline & & 1.4659 & 0.000515 & 0.000432 & 0.005499 \\
\hline \multirow{4}{*}{$\mathrm{F}_{1}$} & \multirow[t]{2}{*}{$(1 \times 2)$} & $50 \cdot 5446$ & $0 \cdot 217005$ & 0.085857 & 0.511369 \\
\hline & & $11 \cdot 9617$ & 0.004204 & 0.003526 & 0.005499 \\
\hline & \multirow[t]{2}{*}{$(2 \times 1)$} & $46 \cdot 4058$ & $0 \cdot 272239$ & 0.157869 & 0.407536 \\
\hline & & $11 \cdot 2322$ & 0.003948 & 0.003311 & 0.005499 \\
\hline \multirow[t]{4}{*}{$\mathrm{F}_{2}$} & \multirow[t]{2}{*}{$(1 \times 2) \times(2 \times 1)$} & $47 \cdot 8880$ & $0 \cdot 274397$ & 0.048750 & $0 \cdot 395750$ \\
\hline & & $1 \cdot 5441$ & 0.000550 & 0.000455 & 0.005499 \\
\hline & \multirow[t]{2}{*}{$(2 \times 1) \times(1 \times 2)$} & $50 \cdot 1658$ & 0.226656 & $0 \cdot 136036$ & 0.390012 \\
\hline & & $6 \cdot 6042$ & 0.002321 & 0.001947 & 0.005499 \\
\hline \multirow{8}{*}{$\mathrm{B}_{1}$} & \multirow[t]{2}{*}{$(1 \times 2) \times(1)$} & $48 \cdot 3511$ & $0 \cdot 280507$ & -0.015107 & \\
\hline & & $5 \cdot 5071$ & 0.001936 & 0.001623 & \\
\hline & \multirow[t]{2}{*}{$(1) \times(1 \times 2)$} & 51.9542 & $0 \cdot 266511$ & $0 \cdot 104881$ & 0.508917 \\
\hline & & $11 \cdot 3921$ & 0.004004 & 0.003358 & 0.005499 \\
\hline & \multirow[t]{2}{*}{$(1) \times(2 \times 1)$} & $50 \cdot 2692$ & 0.238082 & 0.054024 & 0.563464 \\
\hline & & $4 \cdot 1386$ & 0.001455 & 0.001220 & 0.005499 \\
\hline & \multirow[t]{2}{*}{$(2 \times 1) \times(1)$} & $48 \cdot 9146$ & $0 \cdot 207458$ & 0.071012 & $0 \cdot 334024$ \\
\hline & & 5.9667 & 0.002097 & 0.001759 & 0.005499 \\
\hline \multirow[t]{8}{*}{$\mathrm{B}_{2}$} & \multirow[t]{2}{*}{$(1 \times 2) \times(2)$} & $45 \cdot 4619$ & $0 \cdot 276306$ & 0.020131 & \\
\hline & & $5 \cdot 3598$ & $0 \cdot 001884$ & 0.001580 & \\
\hline & \multirow[t]{2}{*}{$(2) \times(1 \times 2)$} & $46 \cdot 6850$ & 0.223464 & $0 \cdot 106178$ & 0.381988 \\
\hline & & 1.9274 & 0.000677 & 0.000658 & 0.005499 \\
\hline & \multirow[t]{2}{*}{$(2) \times(2 \times 1)$} & $46 \cdot 5742$ & 0.232285 & 0.111678 & 0.303786 \\
\hline & & 0.6455 & 0.000227 & 0.000190 & 0.005499 \\
\hline & \multirow[t]{2}{*}{$(2 \times 1) \times(2)$} & $44 \cdot 8404$ & 0.316090 & 0.203333 & 0.442405 \\
\hline & & 3.9772 & 0.001397 & 0.001172 & 0.005499 \\
\hline
\end{tabular}


are most commonly defined as the net additive deviation [d] and the net dominance deviation [h] and refer to the balance of these effects over all loci which contribute to the character (Jinks and Morley Jones, 1958). The variance weighted least squares procedure of Mather and Jinks (1982) was employed in estimating the genetic parameters from the generation means.

\section{RESULTS}

Four competitive parameters, namely the $e$-value, intra-genotypic effect $\left(C_{x x}\right)$, inter-genotypic sensitivity $\left(C_{x y}\right)$ and inter-genotypic pressure $\left(C_{y x}\right)$ were measured for each of the six basic generations as shown in table 1. For each generation, the fit of the linear regression model to the observed data was found to be adequate by comparing the residual variation around the regression lines with the replicate error variance as described by Mather and Caligari, (1981).

Among the fourteen generations assayed (2 parental, $2 F_{1}, 2 F_{2}, 4 B_{1}$ and $4 B_{2}$ ), two of the backcrosses showed an aberrant behaviour. Both of these involved the $F_{1}(1 \times 2)$ that is, flies derived from the cross $(\mathrm{T} 25 \times \mathrm{T} 1)$ as the female parent, although in one case the male parent was T1 and in the other it was T25 (table 1). Each of these backcrosses was characterised by the almost complete competitive exclusion of the $y^{2}$ tester genotype. In fact, only one yellow marked individual emerged from these backcross cultures and this came from one of the duplicate duocultures initiated with ninety yellow and thirty wild type individuals. Such a situation tends to suggest very high aggression of the wild type genotype and also a limited response to the yellow genotype's pressure. However, given the complete absence of yellow survivors, it was not possible to carry out a regression analysis involving the tester genotype $y^{2}$ as indicator. Thus, estimates of inter-genotypic pressure $\left(C_{y x}\right)$ are absent from the relevant backcrosses in table 1 .

\section{(a) The e-value and intra-genotypic competition $\left(C_{x x}\right)$}

A comparison between reciprocal crosses in the $F_{1}, F_{2}, B_{1}$ and $B_{2}$ generations revealed no significant differences. Consequently, all $e$ and $C_{x x}$ estimates for these generations were pooled, as were their error variances. The components of the generation means were estimated using variance weighted least squares, yielding the parameters in
Table 2 Estimates of the genetic parameters and their standard errors together with tests of the significance of their deviation from zero for the competitive parameters $e$ (survival at the reference density) and $C_{x x}$ (intra-genotypic competition)

\begin{tabular}{|c|c|c|}
\hline \multirow{2}{*}{$\begin{array}{l}\text { Genetic } \\
\text { component }\end{array}$} & \multicolumn{2}{|c|}{ Competitive parameter } \\
\hline & $e$ & $C_{x x}$ \\
\hline $\begin{array}{l}m \\
{[d]} \\
{[h]}\end{array}$ & $\begin{array}{r}47 \cdot 3408 \pm 0 \cdot 9639^{* * * *} \\
7 \cdot 1109 \pm 1 \cdot 2836^{* * *}\end{array}$ & $\begin{array}{r}0.3412 \pm 0.0205^{* * *} \\
-0.1478 \pm 0.0499^{* * *}\end{array}$ \\
\hline$\chi_{[4]}^{2}$ & $5 \cdot 8627 \mathrm{~ns}$ & $6 \cdot 1115 \mathrm{~ns}$ \\
\hline
\end{tabular}

The genetic components are defined in the text. The $\chi^{2}$ values test the fit of the additive/dominance model for each of the two competitive parameters. Levels of significance are defined as ns, $P>0.05$; ${ }^{*}, 0.05>P>0.01$; **, $0.01>P>0.001$; ***, $P<0.001$.

table 2. The fit of the model was tested by comparing observed and predicted generation means and the values obtained (table 2) indicate that simple additive and dominance parameters provide an adequate description of the control of these competitive characters. Inspection of the genetic parameters shows that the $e$-value appears to be solely under additive genetic control whereas intragenotypic competition has a significant dominance component. The absence of a significant net additive effect [d] for $C_{x x}$ was not unexpected since the two parents (T25 and T1) do not differ significantly with respect to intra-genotypic competition although they do show substantial differences for the other competitive parameters. The presence of dominance and even a tendency towards heterosis is evident from the observed intragenotypic competitive values for the parental and $F_{1}$ generations shown in table 1 .

\section{(b) Inter-genotypic sensitivity $\left(C_{x y}\right)$}

A comparison between reciprocal crosses revealed no significant differences in the $F_{1}$ or $F_{2}$ generations. There were, however, significant reciprocal differences between those backcrosses in which the $F_{1}(1 \times 2)$ was the maternal parent and those in which the maternal parent was an inbred line. This would indicate a superiority of the $F_{1}$ as mother compared with the inbred as mother as described by Barnes $(1968 a)$. Closer inspection of table 1 shows that in both cases where the $F_{1}$ genotype $(1 \times 2)=(\mathrm{T} 25 \times \mathrm{T} 1)$ was used as the maternal parent, the inter-genotypic sensitivity $\left(C_{x y}\right)$ was substantially reduced in comparison with the reciprocal cross. Thus, the effect of this maternal 
Table 3 Coefficients showing the predicted contribution to the generation means on an additivedominance model in the presence of maternal effects. The genetic components are $\mathrm{m}$, the midhomozygote; $\left[d_{p}\right]$, the additive component of the progeny's own genotype; $\left[h_{p}\right]$, the dominance component of the progeny's own genotype; $\left[h_{m 1}\right]$, the dominance component of the $F_{1}(1 \times 2)$ maternal genotype; $\left(h_{m 2}\right]$, the dominance component of the $F_{1}(2 \times 1)$ maternal genotype; $\left.j\right]$, the additive $\times$ dominance interaction and $[l]$, the dominance $\times$ dominance interaction

\begin{tabular}{|c|c|c|c|c|c|}
\hline \multirow{2}{*}{$\begin{array}{l}\text { Generation } \\
P_{1}\end{array}$} & \multicolumn{2}{|l|}{ Cross } & \multicolumn{3}{|c|}{ Predicted contribution to generation mean } \\
\hline & $(1 \times 1)$ & $m$ & $+\left[d_{p}\right]$ & & \\
\hline $\mathbf{P}_{2}$ & $(2 \times 2)$ & $m$ & $-\left[d_{p}\right]$ & & \\
\hline \multirow[t]{2}{*}{$F_{1}$} & $(1) \times(2)$ & $m$ & $+\left[h_{p}\right]$ & & $+[l]$ \\
\hline & $(2) \times(1)$ & $m$ & $+\left[h_{p}\right]$ & & $+[l]$ \\
\hline \multirow[t]{2}{*}{$\mathrm{F}_{2}$} & $(1 \times 2) \times(2 \times 1)$ & $m$ & $+\frac{1}{2}\left[h_{p}\right]+\left[h_{m 1}\right]$ & & $+\frac{1}{4}[l]$ \\
\hline & $(2 \times 1) \times(1 \times 2)$ & $m$ & $+\frac{1}{2}\left[h_{p}\right]$ & $+\left[h_{m 2}\right]$ & $+\frac{1}{4}[l]$ \\
\hline \multirow[t]{4}{*}{$\mathrm{B}_{1}$} & $(1 \times 2) \times(1)$ & $m$ & $+\frac{1}{2}\left[d_{p}\right]+\frac{1}{2}\left[h_{p}\right]+\left[h_{m 1}\right]$ & & ]$+\frac{1}{4}[l]$ \\
\hline & $(1) \times(1 \times 2)$ & $m$ & $+\frac{1}{2}\left[d_{p}\right]+\frac{1}{2}\left[h_{p}\right]$ & & ]$+\frac{1}{4}[l]$ \\
\hline & $(1) \times(2 \times 1)$ & $m$ & $+\frac{1}{2}\left[d_{p}\right]+\frac{1}{2}\left[h_{p}\right]$ & & ]$+\frac{1}{4}[l]$ \\
\hline & $(2 \times 1) \times(1)$ & $m$ & $+\frac{1}{2}\left[d_{p}\right]+\frac{1}{2}\left[h_{p}\right]$ & $+\left[h_{m 2}\right]$ & ]$+\frac{1}{4}[l]$ \\
\hline \multirow[t]{4}{*}{$\mathrm{B}_{2}$} & $(1 \times 2) \times(2)$ & $m$ & $-\frac{1}{2}\left[d_{p}\right]+\frac{1}{2}\left[h_{p}\right]+\left[h_{m 1}\right]$ & & ]$+\frac{1}{4}[l]$ \\
\hline & $(2) \times(1 \times 2)$ & $m$ & $-\frac{1}{2}\left[d_{p}\right]+\frac{1}{2}\left[h_{p}\right]$ & & ]$+\frac{1}{4}[l]$ \\
\hline & $(2) \times(2 \times 1)$ & $m$ & $-\frac{1}{2}\left[d_{p}\right]+\frac{1}{2}\left[h_{p}\right]$ & & ]$+\frac{1}{4}[l]$ \\
\hline & $(2 \times 1) \times(2)$ & $m$ & $-\frac{1}{2}\left[d_{p}\right]+\frac{1}{2}\left[h_{p}\right]$ & $+\left[h_{m 2}\right]$ & ]$+\frac{1}{4}[l]$ \\
\hline
\end{tabular}

genotype is to improve competitive ability by reducing inter-genotypic sensitivity. This effect is revealed as a significant difference in the backcrosses, but not in the $F_{2}$. In view of this $F_{1}$ maternal effect an alternative genetical model (proposed by Barnes, 1968a) was employed (table 3 ). This attempts to separate the effects of the progeny's own genotype from those of the maternal genotype and to assess the magnitude of the additive and dominance components of the maternal genotype on inter-genotypic sensitivity $\left(C_{x y}\right)$.

Each of the components in table 3 were estimated using a variance weighted least squares procedure and the estimates, together with their standard errors and tests of their deviation from zero are given in table 4. Clearly, the model is shown to provide a perfectly adequate description of the genetical control of inter-genotypic sensitivity. The absence of a significant dominance component of the maternal genotype $\left[d_{m}\right]$ was not unexpected since the $F_{1}$ reciprocals were not significantly different. There was, however, dominance in the direction of reduced inter-genotypic sensitivity (superior competitive ability) both for the genes dispersed between the two parents $\left[h_{p}\right]$ and for those reflecting the $F_{1}(1 \times 2)$ maternal genotype $\left[h_{m 1}\right]$. A comparison of the magnitude of the components shows a high level of heterosis determined by the progeny's own genotype $\left(\left[h_{p}\right]>\left[d_{p}\right]\right)$ and also by the $F_{1}(1 \times 2)$ maternal genotype $\left(\left[h_{m 1}\right]>\right.$ $\left.\left[d_{m}\right]\right)$. Furthermore, all heterosis is directed towards reduced inter-genotypic sensitivity or, in other words, a superior competitive ability.

Among the non-allelic interactions both $[j]$ (additive $\times$ dominance interactions) and $[l]$ (dominance $\times$ dominance interactions) were significant (table 4$)$. The opposite sign of $[l] \mathrm{com}$ pared to $\left[h_{p}\right]$ suggests that any heterosis due to the progeny's own genotype is of minor importance, since it is determined as $\left[h_{p}\right]+[l]>\left[d_{p}\right]+[i]$ (Mather and Jinks, 1982).

Table 4 Estimates of the genetic components defined in table 3 together with their standard errors and $t$-tests for the deviation of each parameter from zero. Levels of significance are as defined in table 2 . The $\chi_{[7]}^{2}$ value is a test of the adequacy of the model and shows no significant difference between observed and predicted generation means

\begin{tabular}{lrll}
\hline $\begin{array}{l}\text { Genetic } \\
\text { component }\end{array}$ & Estimate & $\begin{array}{l}\text { Standard } \\
\text { error }\end{array}$ & Significance \\
\hline$m$ & 0.213517 & 0.029715 & $* * *$ \\
{$\left[d_{p}\right]$} & -0.131446 & 0.029715 & $* * *$ \\
{$\left[h_{p}\right]$} & -0.424811 & 0.113689 & $* *$ \\
{$\left[h_{m 1}\right]$} & -0.047335 & 0.022204 & $*$ \\
{$\left[h_{m 2}\right]$} & 0.057915 & 0.026287 & $*$ \\
{$[j]$} & 0.153993 & 0.077466 & $*$ \\
{$[l]$} & 0.334289 & 0.116743 & $*$ \\
\hline$\chi_{[7]}^{2}=12.144$ & $P>0.05$ & ns & \\
\hline
\end{tabular}




\section{(c) Inter-genotypic pressure $\left(C_{y x}\right)$}

The emergence of only one yellow fly from the backcross duocultures having the maternal parent $F_{1}(1 \times 2)=(T 25 \times T 1)$ suggests maternal heterosis as described for inter-genotypic sensitivity. However, because of the absence of a duoculture regression line for these cultures it was not possible to analyse the data using the genetic model proposed in table 3 . Consequently, it was decided to omit the two backcrosses from which no yellow flies emerged and to fit a simplified additivedominance model to the remaining twelve crosses. Estimates of inter-genotypic pressure $\left(C_{y x}\right)$ for each cross in each of the generations, $\mathrm{P}_{1}, \mathrm{P}_{2}, \mathrm{~F}_{1}$, $F_{2}, B_{1}$ and $B_{2}$ are set out in table 1 . As found in the previous analysis of inter-genotypic sensitivity, no significant reciprocal differences were evident in the $F_{1}$ and $F_{2}$ generations for inter-genotypic pressure. There were, however, significant differences between the backcross reciprocals which could not, therefore, be pooled. The components of the generation means were estimated as before, using a variance weighted least squares procedure and the parameter estimates, their standard errors and tests of their deviation from zero are given in table 5. All of these parameters were statistically very highly significant with the net additive and net dominance effects of relatively similar magnitude. The fit of this model was determined by comparing observed and predicted generation means, yielding $\chi_{[9]}^{2}=9 \cdot 156 ; P>0 \cdot 05$. Thus, there is no reason to suspect anything other than additive and dominance effects in the control of intergenotypic pressure. There is some evidence for heterosis in the control of this competitive parameter, given that $[h]>[d]$ and this heterosis is directed towards an increase in inter-genotypic pressure or, in other words, towards a competitively superior phenotype. The presence of

Table 5 Estimates of the mid-homozygote $m$ and the net additive $[d]$ and dominance $[h]$ components of the generation means for inter-genotypic pressure, together with their standard errors. Results of $t$-tests for the deviation of each parameter from zero are given with significance levels as defined in table 2 . The $\chi^{2}$ value tests the adequacy of the model and shows no significant difference between the observed and predicted generation means

\begin{tabular}{llll}
\hline $\begin{array}{l}\text { Genetic } \\
\text { component }\end{array}$ & Estimate & $\begin{array}{l}\text { Standard } \\
\text { error }\end{array}$ & Significance \\
\hline$m$ & 0.321838 & 0.042813 & $* * *$ \\
{$[d]$} & $0 \cdot 117944$ & 0.039637 & $* * *$ \\
{$[h]$} & 0.162012 & 0.040250 & $* * *$ \\
\hline$\chi_{[9]}^{2}=9.156$ & $P=0.50-0.10$ & ns & \\
\hline
\end{tabular}

heterosis was not unexpected since the $F_{1}$ can be seen to exceed the $P_{1}$ in table 1 . Since there is no evidence for any significant non-allelic interaction it could be that the observed heterosis is due to either over-dominance at some or all of the loci involved, or dispersion of the dominant alleles between the parents.

\section{DISCUSSION}

Any interpretation of the results obtained in this experiment must be based on the assumption that the environmental conditions remained constant during the determination of all parameters. The experimental design involved raising all cultures simultaneously within a single individually randomised block. This will, of course, minimise the impact of any common environmental fluctuations. However, because of the nature of the competitive parameters under investigation, it is not possible totally to negate the role of the environment. For example, both competitive sensitivity and pressure are determined partly by the environment created by the competitors themselves, through the processes of interference and detoxification (Hemmat and Eggleston, 1988b; Bottella et al., 1985). In addition, it must be remembered that the determination of genetic effects is dependent upon the amount of competitive stress or selective pressure. This is itself determined by a wide and complex range of parameters, including larval feeding rates and conversion efficiencies (de Miranda and Eggleston, $1988 a, b)$ and the interference imposed by the contemporary competitors (Hemmat and Eggleston, 1988b). Consequently any alteration to the competitive environment may have a profound effect on the estimation of the genetic parameters.

All of the models employed in these experiments were designed assuming the absence of sex linkage. This appears to be a reasonable assumption in the case of the competitive parameters investigated here. For example, no significant reciprocal differences in the $F_{1}$ or $F_{2}$ generations were observed for any of the four parameters studied. Moreover, other recent investigations also suggest an absence of sex-specific competitive effects. The chromosome assay of Caligari and Mather (1988) showed that no main effect on the control of either aggression or response could be localised to the $\mathrm{X}$-chromosome. This may indeed be a pre-requisite for stability of the sex-ratio under intense selection pressure. Clearly, even a minor competitive superiority conferred by the $\mathrm{X}$ - 
chromosome could ultimately lead to a pronounced excess of females.

Of the four competitive parameters investigated here, the role of one of them (e-value) in the process of competition is not clear. Caligari and Mather (1988) have questioned whether the $e$ value can be considered as a competitive parameter. It is, for example, based on a static rather than a dynamic measurement, taken at a single density (the reference density). All other competitive parameters are measured as a rate of change in some character over a series of densities and their dynamic nature is quite clear. For these reasons, the $e$-value presumably reflects agencies which have an effect on the fitness of a genotype but which are not necessarily dependent upon population density. The interpretation of the $e$ value favoured in these investigations is that it reflects the basal competitive characteristics of the indicator genotype and provides a datum from which the other competitive parameters may be measured. The experiments reported here show that the $e$-value is under simple additive genetic control with no evidence of any significant net dominance effect.

Intra-genotypic competition $\left(C_{x x}\right)$ was found to exhibit a significant net dominance effect $[h]$ with no evidence of significant additive deviations. The observed values in the $F_{1}$ and parental generations suggest a high level of dominance and a tendency towards heterosis. The absence of any significant net additive effect $[d]$ was expected, since the two parents did not differ significantly in their intra-genotypic competition. However, it is possible that two different parental genotypes would reveal an additive component to the control of this parameter. This assumption is supported by de Miranda and Eggleston (1988c) who found intra-genotypic competition to have a substantial additive component.

The significant genetic components controlling inter-genotypic sensitivity $\left(C_{x y}\right)$ were additive and dominance components of the progeny's own genotype $\left(\left[d_{p}\right]\right.$ and $\left.\left[h_{p}\right]\right)$, and dominance components of the $F_{1}$ maternal genotypes ( $\left[h_{m 1}\right]$ and $\left.\left[h_{m 2}\right]\right)$, as well as additive $\times$ dominance non-allelic interactions $[j]$ and dominance $\times$ dominance nonallelic interactions $[l]$. Heterosis was found to be determined by the progeny's own genotype $\left[h_{p}\right]>$ $\left[d_{p}\right]$, and by the $F_{1}(1 \times 2)$ maternal genotype $\left[h_{m 1}\right]>\left[d_{m}\right]$. The principle components were dominance in the direction of low inter-genotypic sensitivity or in other words, competitive superiority. Moreover, since [l] was of opposite sign to $\left[h_{m 1}\right]$ and $\left[h_{p}\right]$ the observed heterosis was most probably due to overdominance or dispersion of dominant increasing alleles in the parental lines, rather than to non-allelic interaction.

Inter-genotypic competitive pressure $\left(C_{y x}\right)$ was found to be controlled by additive and dominance effects of approximately equal magnitude. Heterosis due to the progeny's own genotype was found to play a part in controlling this parameter as shown by the relationship $\left(\left[h_{p}\right]>\left[d_{p}\right]\right)$. Moreover, heterosis determined by the $F_{1}$ maternal genotype cannot be ignored, especially in those backcrosses involving the $F_{1}$ maternal genotype $(\mathrm{T} 25 \times \mathrm{T} 1)$. The almost complete absence of emerging yellow marked flies in these two backcrosses suggests a high level of inter-genotypic pressure due to $F_{1}$ maternal heterosis. However, the absence of yellow flies prevented the estimation of this quantity through regression analysis. Consequently, the genetic analysis was of necessity restricted by excluding these two backcrosses. The results do not, therefore, provide any information about the maternal effect components. The analysis did, however, reveal significant net additive and net dominance effects although no non-allelic interactions were detected. In the present investigation it is difficult to say whether the observed heterosis is determined by dispersion of dominant genes or by overdominance. Given that there are few recorded examples of overdominance for major Mendelian loci, current opinion would favour the hypothesis of heterosis through gene dispersion. The extra vigour of the $F_{1}$ hybrid recorded in these experiments may be the manifestation of a superior gene array, reflecting increased heterozygosity following dispersion of the increasing alleles between the inbred parents. This is supported by the absence of non-allelic interactions which can also contribute to heterosis.

The final point concerns the type of genetical architecture displayed by these competitive parameters. The two main competitive parameters, inter-genotypic pressure and sensitivity were governed by systems showing high levels of heterosis towards improved yield. This type of genetical architecture may be related to the forces of natural selection to which the characters have been subjected. Fisher $(1928 a, b, 1929,1930)$ postulated that where two alleles exist together in a population, natural selection will favour those heterozygotes whose phenotypic expression is changed, by modifiers, towards the phenotype of the homozygote carrying the more favourable allele. This interpretation may be extended to include characters governed by polygenic systems. In this way Mendelian populations may be said 
to possess the property of genetic homeostasis, defined as the tendency to maintain a genetic composition that maximises fitness for the environment in which the population lives. Genetic homeostasis may involve natural selection for intermediate rather than extreme phenotypes and one possible mechanism for this regulation of genetic composition is superiority of heterozygotes with respect to fitness. It may be argued that the only important function of heterozygosity is that it permits segregation to occur, thus providing a population with the genetic flexibility to adapt. Genetic homeostasis, which is based on the superiority of heterozygotes, has been proposed as a form of architecture which may ensure the maintenance of genetic variation in natural populations (Lewontin, 1955) and which implies a situation of stabilizing selection. However, it is also possible and perhaps more realistic to interpret our results in terms of directional selection. Ultimately, this would lead to the evolution of unidirectional dominance, together with duplicate gene interactions as described by Barnes $(1968 b)$. Indeed, this is what we find for inter-genotypic sensitivity. This type of architecture has been shown to maintain uniformly high levels of fitness within Drosophila populations for a variety of characters including viability (Breese and Mather, 1960); hatchability, egg production and egg-pupal survival (Keller and Mitchell, 1964) and hatchability (Kearsey, 1965). It now remains to be seen whether, by using similar forms of analysis, this type of genetical architecture can be confirmed for competitive parameters in organisms other than Drosophila.

Acknowledgements We are grateful to Dr J. R. de Miranda for his thoughts and advice, to Lynda Mitchell for technical assistance and to Hilda Longworth for preparing the manuscript. Financial assistance was provided by the Lister Institute of Preventive Medicine (Fellowship to PE) and the Iranian Ministry of Education.

\section{REFERENCES}

BARNES, B. W. 1968a. Stabilising selection in Drosophila melanogaster. Heredity, 23, 433-442.

BARNES, B. W. $1968 \mathrm{~b}$. Maternal control of heterosis for yield in Drosophila melanogaster. Heredity, 23, 563-572.

BONNIER, G. 1961. Experiments on hybrid superiority in Drosophila melanogaster. I. egg laying capacity and larval survival. Genetics, 46, 9-24.

BotTella, L. M., MOYA, A., GONZALEZ, C. AND MERVA, J. L. 1985. Larval stop, delayed development and survival in overcrowded cultures of Drosophila melanogaster: effect of urea and uric acid. J. Insect. Physiol., 31, 179-185.
BREESE, E. L. AND MATHER, K. 1960. The organisation of polygenic activity within a chromosome in Drosophila: II. Viability. Heredity, 14, 375-399.

CALIGARI, P. D. S. AND MATHER, K. 1988. Competitive interactions in Drosophila melanogaster: IV. Chromosome assay. Heredity, 60, 355-366.

DE MIRANDA, J. R. AND EGGLESTON, P. 1988a. Larval competition in Drosophila melanogaster: 1. Estimation of larval growth parameters. Heredity, 60, 205-212.

DE MIRANDA, J. R. AND EGGleston, P. 1988b. Larval competition in Drosophila melanogaster: II. Comparing biological and competitive parameters. Heredity, 60 , 213-219.

DE MIRANDA, J. R. AND EGgleston, P. 1988c. Genetic analysis of larval competition in Drosophila melanogaster. Heredity, 61, 339-346.

EGGLESTON, P. 1985. Variation for aggression and response in the competitive interactions of Drosophila melanogaster. Heredity, 54, 43-51.

FALCONER, D. S. 1986. Introduction to Quantitative Genetics (2nd edn). Longman Scientific and Technical, New York.

FISHER, R. A. 1928a. The possible modification of the response of the wild type to recurrent mutations. Amer. Nat., 62, $115-126$.

FISHER, R. A. 1928 b. Two further notes on the origin of dominance. Amer. Nat., 62, 571-574.

FISHER, R. A. 1929. The evolution of dominance; reply to Professor Sewall Wright. Amer. Nat., 63, 553-556.

FISHER, R. A. 1930. The Genetical Theory of Natural Selection. Clarendon Press, Oxford.

FISHER, R. A., IMMER, F. R. AND TEDIN, O. 1932. The genetical interpretation of statistics of the third degree in the study of quantitative inheritance. Genetics. 17, 107-124.

FISHER, T. A. AND YATES, F. 1963. Statistical Tables for Biological, Agricultural and Medical Research (6th edn). Oliver and Boyd, Edinburgh.

HEMMAT, M. AND EGGleston, P. 1988a. Competitive interactions in Drosophila melanogaster: recurrent selection for aggression and response. Heredity, 60, 129-137.

HEMMAT, M. AND EGGleston, P. 1988b. Competitive interactions in Drosophila melanogaster: genetic variation for interference through media conditioning. Heredity, 61, 347-354.

JINKS, J. L. AND MORLEY JONES, R. 1958. Estimation of the components of heterosis. Genetics, 43, 223-234.

KEARSEY, M. J. 1965. The interaction of competition and food supply in two lines of Drosophila melanogaster. Heredity, $20,169-181$.

KELLER, E. C. AND MITCHELL, D. F. 1964. Inter-chromosomal genotypic interactions in Drosophila: II. An analysis of viability characters. Genetics, 49, 293-307.

LERNER, I. M. AND HO, F. K. 1962. Genotype and competitive ability of Tribolium species. Amer. Nat., 95, 329-343.

LEWONTIN, R. C. 1955 . The effects of population density and composition on viability in Drosophila melanogaster. Evolution, 9, 27-41.

LINNEY, R., BARNES, B. W. AND KEARSEY, M. J. 1971. Variation for metrical characters in Drosophila populations. Heredity, 27, $163-174$.

MATHER, K. 1983. Response to selection. In Ashburner, M., Carson, H. L. and Thompson, J. N. Jnr (eds) The Genetics and Biology of Drosophila. vol. 3c Academic Press, pp. $152-215$.

MATHER, K. AND COOKE, P. 1962. Differences in competitive ability between genotypes of Drosophila. Heredity, 17, 381-407. 
MATHER, K. AND CAlGaRI, P. D. S. 1981. Competitive interactions in Drosophila melanogaster: II. Measurement of competition. Heredity, 46, 239-254.

MATHER, K. AND JINKS, J. L. 1982. Biometrical Genetics (3rd edn). Chapman and Hall, London.

MATHER, K. AND CAligari, P. D. S. 1983. Pressure and response in competitive interactions. Heredity, 51, 435-454.

SPITTERS, C. J. T. 1983. An alternative approach to the analysis of mixed cropping experiments. I. Estimation of competition effects. Neth. J. Agric. Sci., 31, 1-11.

SUEHIRO, K. AND OGAWA, H. 1980. Competition between two annual herbs, Atriplex gmelini: C. A. Mey and Chenopodium album L. in mixed cultures irrigated with sea-water of various concentrations. Oecologia, 45, 167177.
WATKINSON, A. R. 1984. Yield density relationships: the influence of resource availability on growth and self-thinning in populations of Vulpia fasciculata. Ann. Bot., 53, 967-976.

WRIGHT, A. J. 1981. The analysis of yield-density relationships in binary mixtures using inverse polynomials. J. Agric. Sci. Camb., 96, 561-567. 